\title{
Computing seismic fragility curves using polynomial chaos expansions
}

\author{
B. SUDRET \& C. V. MAI \\ ETH Zürich, Institute of Structural Mechanics, Chair of Risk, Safety \& Uncertainty Quantification \\ Wolfgang-Pauli-Strasse 15, CH-8093 Zürich \\ email: sudret@ibk.baug.ethz.ch
}

\begin{abstract}
Fragility curves are commonly used in civil engineering to estimate the vulnerability of structures to earthquakes. The probability of failure associated with a failure criterion (e.g. the maximal inter-storey drift being greater than a prescribed threshold) is represented as a function of the intensity of the earthquake (e.g. peak ground acceleration or spectral acceleration). Computing fragility curves relies upon running a large number of transient dynamic (possibly non linear) analyses using a set of synthetic or measured ground motions. In this paper we introduce sparse polynomial chaos expansions as a way to reduce the associated computational burden. Non stationary synthetic ground motions are generated using a method recently proposed by Rezaeian \& Der Kiureghian, which is based on modulating a filtered Gaussian white noise process. Sparse PC expansions of the structural response are computed from a relatively small (e.g. 1,000) set of accelerograms and post-processed in order to evaluate the fragility curves. The approach is compared to the classical curves derived under the assumption of log-normality. For linear structures the lognormal assumption is validated by our calculations and the classical approach is improved using some analytical derivations. The various approaches are illustrated on a typical 3-storey building using the finite element software OpenSees.
\end{abstract}

\section{INTRODUCTION}

Fragility curves are commonly used in seismic probabilistic risk assessment in order to estimate the vulnerability of structures to earthquakes, e.g. the probability that a structure fails to fulfil a safety criterion during the ground motions. The probability of failure associated with a failure criterion (e.g. the maximal inter-storey drift being greater than a prescribed threshold) is represented as a function of the intensity of the earthquake (e.g. peak ground acceleration (PGA) or spectral acceleration (SA)) [7]. Fragility curves have become an increasingly popular tool in the recent years for assessing the safety of various types of structures such as nuclear power plants [18], bridges [5], buildings [17] or wind turbines.

From the mathematical point of view, a fragility curve represents the conditional probability that a prescribed threshold is exceeded given the intensity of an earthquake. The classical approach to compute fragility curves consists in assuming that the curves have a lognormal shape [7, 18], and their parameters are determined by linear regression. This assumption can ease the estimation of the curves, but also leads to a large epistemic uncertainty when used with a limited set of data.

The computation of fragility curves based on
Monte Carlo simulation (e.g. Incremental Dynamic Analysis (IDA) in Vamvatsikos and Cornell [16]) requires a large number of transient dynamic analysis of the structure under seismic excitations, that are either recorded or synthetic. Due to the lack of recorded signals with the properties of interest (e.g. magnitude, duration, etc.), it is common for researchers to generate suitable samples of synthetic earthquakes. Both the generation of a large number of signals and the transient dynamic analysis of the structure subject to them are highly time-consuming. Sparse polynomial chaos expansions (PCE) [3, 4] is therefore an attractive option to limit the number of transient analysis and to better take advantage of the results. In this paper we demonstrate the use of PC expansions to establish fragility curves without assuming a particular shape. The paper is organized as follows: In Section 2, the approach recently proposed by Rezaeian $\&$ Der Kiureghian to generate synthetic earthquakes is briefly recalled. Then the principle of PCE is described in Section 3. In Section 4, the fragility curves obtained with and without PCE are compared, and a contribution to classical "lognormal"approach is proposed on the basis of the obtained results. 


\section{SEISMIC GENERATION}

In this section, we summarize the parameterized approach proposed by Rezaeian and Der Kiureghian [13] in order to simulate synthetic ground motions. The seismic acceleration is represented as a nonstationary process. Der Kiureghian and Rezaeian separate the non-stationarity into two components, namely a spectral and a temporal one, by means of a modulated filtered Gaussian white noise:

$a(t)=\frac{q(t, \boldsymbol{\alpha})}{\sigma_{h}(t)} \int_{-\infty}^{t} h[t-\tau, \boldsymbol{\lambda}(\tau)] \omega(\tau) \mathrm{d} \tau$

in which $q(t, \boldsymbol{\alpha})$ is the deterministic non-negative modulating function and the quantity inside the brackets is the stationary response of a linear filter subject to a Gaussian white noise excitation.

The Gaussian white-noise process denoted by $\omega(\tau)$ will pass a filter $h[t-\tau, \boldsymbol{\lambda}(\tau)]$ which is selected as an impulse-response function:

$$
\begin{aligned}
& h[t-\tau, \boldsymbol{\lambda}(\tau)]=0 \text { for } t<\tau \\
& h[t-\tau, \boldsymbol{\lambda}(\tau)]=\frac{\omega_{f}(\tau)}{\sqrt{1-\zeta_{f}^{2}(t)}} \exp \left[-\zeta_{f}(\tau) \omega_{f}(\tau)(t-\tau)\right]
\end{aligned}
$$$$
\times \sin \left[\omega_{f}(\tau) \sqrt{1-\zeta_{f}^{2}(\tau)}(t-\tau)\right] \quad \text { for } \quad t \geq \tau
$$

where $\boldsymbol{\lambda}(\tau)=\left(\omega_{f}(\tau), \zeta_{f}(\tau)\right)$ is the vector of timevarying parameters of the filter $h . \omega_{f}(\tau)$ and $\zeta_{f}(\tau)$ are the filter's natural frequency and damping ratio at instant $\tau$, respectively. They represent the evolving predominant frequency and bandwidth of the ground motion. The statistical analysis of real signals shows that the $\zeta_{f}(\tau)$ may be taken as a constant $\left(\zeta_{f}(\tau) \equiv \zeta\right)$ while the predominant frequency varies linearly in time:

$\omega_{f}(\tau)=\omega_{m i d}+\omega^{\prime}\left(\tau-t_{\text {mid }}\right)$

in which $t_{\text {mid }}$ is the instant at which $45 \%$ of the expected Arias intensity $I_{a}$ is reached, $\omega_{\text {mid }}$ is the filter's frequency at instant $t_{m i d}$ and $\omega^{\prime}$ is the slope of linear evolution. After being normalized by the standard deviation $\sigma_{h}(t)$, the integral in Eq. (1) becomes a unit variance process with time-varying frequency and constant bandwidth.

The non-stationarity in intensity is then captured by the modulated function $q(t, \boldsymbol{\alpha})$. This time-modulating function determines the shape, intensity and duration $T$ of the signal. A Gamma-like function is usually used:

$q(t, \boldsymbol{\alpha})=\alpha_{1} t^{\alpha_{2}-1} \exp \left(-\alpha_{3} t\right)$ where $\boldsymbol{\alpha}=\left(\alpha_{1}, \alpha_{2}, \alpha_{3}\right)$ is directly related to the following characteristics of the signal, namely the expected Arias intensity $I_{a}$, the duration of the strong motion phase $D_{5-95}$ between the instants at which the $5 \%$ and $95 \%$ of $I_{a}$ are reached, and the instant $t_{\text {mid }}$.

The acceleration in Eq. (1) can be discretized as follows:

$\hat{a}(t)=q(t, \boldsymbol{\alpha}) \sum_{i=1}^{n} s_{i}\left(t, \boldsymbol{\lambda}\left(t_{i}\right)\right) U_{i}$

where the standard normal random variable $U_{i}$ represents an impulse at instant $t_{i}=i \times \frac{T}{n}, i=1, \ldots, n$, ( $\mathrm{T}$ is the total duration) and $s_{i}\left(t, \boldsymbol{\lambda}\left(t_{i}\right)\right)$ is given by:

$$
s_{i}\left(t, \boldsymbol{\lambda}\left(t_{i}\right)\right)=\frac{h\left[t-t_{i}, \boldsymbol{\lambda}\left(t_{i}\right)\right]}{\sqrt{\sum_{j=1}^{i} h^{2}\left[t-t_{j}, \boldsymbol{\lambda}\left(t_{j}\right)\right]}}
$$

As a summary, the proposed model consists of 3 temporal parameters $\left(\alpha_{1}, \alpha_{2}, \alpha_{3}\right), 3$ spectral parameters $\left(\omega_{m i d}, \omega^{\prime}, \zeta_{f}\right)$ and the standard Gaussian random vector $\boldsymbol{U}$ of size $n$.

In this study, we generated the accelerograms with deterministic parameters $\left\{\alpha_{1}, \alpha_{2}, \alpha_{3}, \omega_{m i d}, \omega^{\prime}, \zeta_{f}\right\}$. However, due to the Gaussian white-noise process $\boldsymbol{U}$, the resulting synthetic signals have different trajectories and effects on the structure.

\section{POLYNOMIAL CHAOS EXPANSIONS}

Consider a mechanical system described by a computational model $\mathcal{M}(\boldsymbol{U})$. In civil engineering, $\mathcal{M}$ is usually a black box program solving a set of partial differential equations, e.g. a finite elements code. Let us assume that the input vector $\boldsymbol{U}$ has $n$ independent input random variables $U_{1}, \ldots, U_{n}$ with prescribed joint probability density function $f_{U}$. Polynomial chaos expansion (PCE) aims at representing the function $\mathcal{M}($.) onto a suitable orthonormal basis $\left\{\psi_{\boldsymbol{\alpha}}(\boldsymbol{U}), \boldsymbol{\alpha} \in \mathbb{N}^{n}\right\}$ as follows:

$\mathcal{M}(\boldsymbol{U})=\sum_{\boldsymbol{\alpha} \in \mathbb{N}^{n}} a_{\boldsymbol{\alpha}} \psi_{\boldsymbol{\alpha}}(\boldsymbol{U})$

In this equation the $\psi_{\boldsymbol{\alpha}}(\boldsymbol{U})$ 's are multivariate orthonormal polynomials (w.r.t the probability measure associated with $f_{\boldsymbol{U}}$ ) and $a_{\boldsymbol{\alpha}}$ 's are the deterministic coefficients to be computed. In the present application the ground motions are simulated using a set of standard normal random variables (Eq.(5)). The associated polynomials are the so-called Hermite polynomials. In practice a truncated series is used, for instance by retaining only those polynomials whose total degree is smaller than or equal to $p$. When using such a truncation scheme the number of unknowns of the problem is:

$P=\frac{(n+p) !}{n ! p !}$ 
when $n$ is the size of the input random vector. The coefficients may be computed by solving a mean-square minimization problem [1]. However the number of unknowns dramatically increases with $n$ and makes such a direct approach intractable for large $n$, which is the case here since earthquake signals need typically a few hundred random variables for an accurate representation.

Fortunately large-dimensional problems often obey the "sparsity of effect" principle meaning that not all input variables have a significant impact on the model output. This idea has lead to the development of sparse polynomial chaos expansions [2, 3]. In particular the least angle regression algorithm (LAR) [8] allows one to find directly the non zero coefficients in a given expansion and leads to sparse solutions. This algorithm may be used with fixed or adaptive experimental designs [3] and with scalar or vector output quantities when used together with principal component analysis [4].

\section{PC EXPANSIONS OF INTER-STOREY DRIFTS}

\subsection{Problem statement}

In this section, we use polynomial chaos expansions together with transient finite element analysis in order to evaluate the fragility curve of a 3-storey 3-span steel frame structure with the following dimensions: storey-height $H=3 \mathrm{~m}$, span-length $L=5 \mathrm{~m}$. The considered frame is supposed to have a linear elastic behavior. The Young's modulus of steel is equal to $E=205,000 \mathrm{MPa}$, and the Poisson's ratio is $\nu=0.3$. The loading consists of dead-load (from the frame elements as well as the supported floor), and variable load in accordance with Eurocode 1.

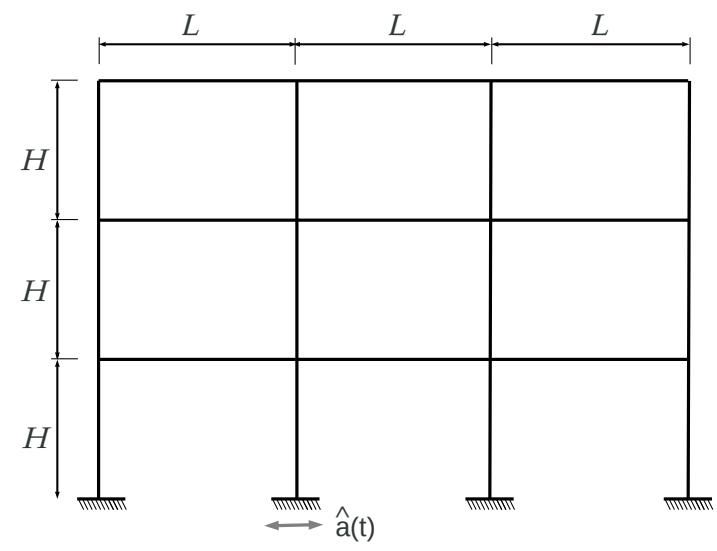

Figure 1: Steel frame structure

The structure is subject to ground motions modeled by the time-history of acceleration at the ground level. According to the model described in Section 2, the following values are used: $\alpha_{1}=4.210^{-4}$, $\alpha_{2}=5.5576, \alpha_{3}=0.3192, \omega_{\text {mid }}=40.05 \mathrm{rad} / \mathrm{s}, \omega^{\prime}=$ $-0.5279 \mathrm{rad} / \mathrm{s}, \zeta_{f}=0.1985$. Each ground motion is modeled by an input vector $\boldsymbol{U}$ made of 500 independent Gaussian random variables $U_{i}$. The resulting signals will have similar spectral content, total duration, shape and effective duration (Figure 2). Using such a representation, the range of the peak ground acceleration (PGA) is from 1.3 to $4.3 \mathrm{~m} / \mathrm{s}^{2}$.

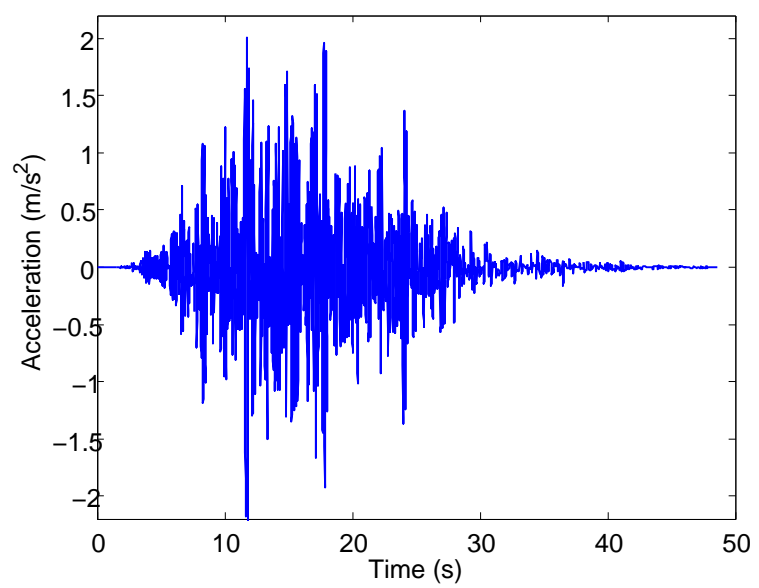

Figure 2: Seismic signal generated from a particular realization of 500 random variables $U_{i}$

In this study, the PGA is used as an intensity measure to plot fragility curves and the admissible maximal inter-storey drift is denoted by $\delta_{0}$. The different values of $\delta_{0}$ define the level of damage that the structure suffers if the maximal inter-storey drift $\Delta$ exceeds the criterion $\delta_{0}$, e.g. slight damage if $\frac{\Delta}{\delta_{0}} \geq \frac{1}{400}$ or severe damage if $\frac{\Delta}{\delta_{0}} \geq \frac{1}{150}$. We first run a set of 1,000 transient dynamic analyses using the finite element code OpenSees [11]. From these 1,000 runs, we built the metamodel of the time-history inter-storey drift $\delta^{k}\left(t_{i}\right)$ for story $k=1,2,3$ at instants $t_{i}$ varying from $5 \mathrm{~s}$ to $30 \mathrm{~s}$ with time step $d t=0.02 \mathrm{~s}$. This leads to the computation of 3753 sparse PC expansions.

Once the PC expansions are obtained, they are post-processed by Monte Carlo simulation using $N=10^{5}$ samples, say $\left\{\underline{u}_{j}, j=1, \ldots, N\right\}$. For each realization, we compute the maximal inter-storey drifts $\Delta\left(\underline{u}_{0}\right)=\max _{k=1,2,3} \max _{t_{i} \in[5,30]}\left|\delta^{k}\left(t_{i}, \underline{u}_{0}\right)\right|$ caused by the ground motions $\hat{a}\left(t, \underline{u}_{0}\right)$. The pairs $\left\{\left(P G A\left(\underline{u}_{j}\right), \Delta\left(\underline{u}_{j}\right)\right), j=1, \ldots, N\right\}$ were collected and postprocessed following different approaches to establish the fragility curves. The data from 1,000 original runs were also used in the classical approach based on a lognormal assumption.

\subsection{PCE for instantaneous response}

Let us consider first the PCE of instantaneous interstorey drifts for the 3 stories at different instants, e.g. $10 \mathrm{~s}, 20 \mathrm{~s}, 30 \mathrm{~s}$. To validate the metamodels, a new set of 50 input vectors $\boldsymbol{U}$ was generated. The corresponding inter-storey drifts were then calculated using both the finite element model and the PC expansion for validation purpose. The results are compared in Figure 3 .

The quality of the PC expansion is measured by the leave-one-out $Q^{2}$ estimate (see [2] for details). Figure 


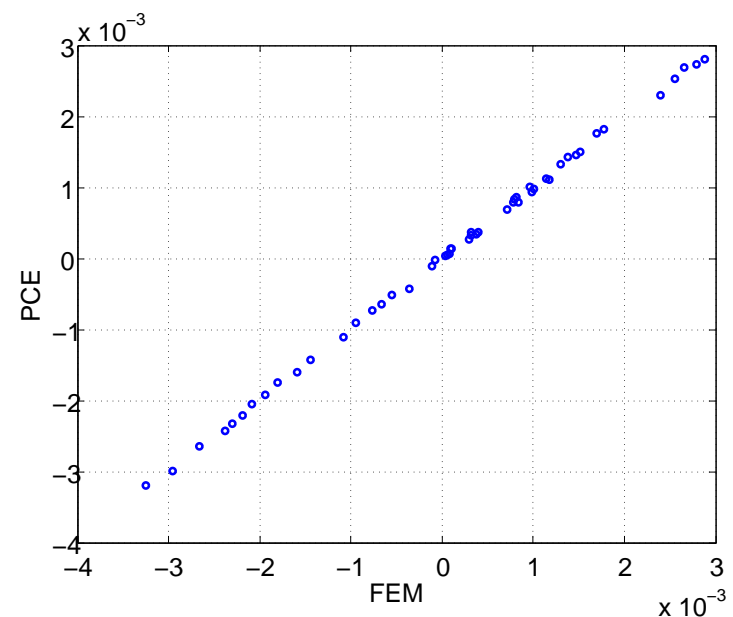

Figure 3: Prediction of the first inter-storey drift at $t=20 \mathrm{~s}$

3 shows the accuracy of the PC metamodel for the first story drift at $\mathrm{t}=20 \mathrm{~s}$. The mean-square error is equal to $1-Q^{2}=5 \cdot 10^{-3}$. The final basis obtained by Least Angle Regression happens to contain first order polynomials only, which already ensure a high accuracy of the approximation. The explanation can be found in Figure 4, the PDF of the instantaneous inter-storey drift is indeed a Gaussian distribution. This explains why the instantaneous drift can be approximated with a first order polynomial function of the input vector $\boldsymbol{U}$. This was expected since the seismic signals have deterministic temporal and spectral content, i.e. at every instant, the signal is a linear combination of Gaussian random variables $U_{i}$, see Eq. (5). As a consequence, the response of a linear system to a zero-mean Gaussian process is a zero-mean Gaussian process. In conclusion, under our current assumptions the instantaneous drift is normally distributed, and the proposed PC expansion scheme perfectly captures this feature.

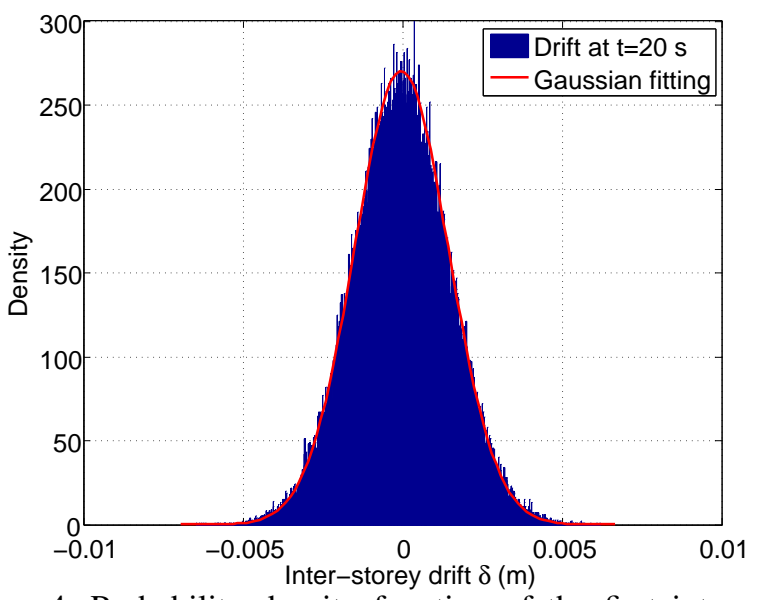

Figure 4: Probability density function of the first inter-storey drift at $\mathrm{t}=20 \mathrm{~s}$ from PCE-based prediction $\left(10^{5}\right.$ samples of the PCE) and Gaussian distribution

\subsection{Sensitivity analysis: structural "memory"}

In this section we investigate the role of each single input random $U_{i}$ variable (used for representing the ground motion) onto the instantaneous drift of the structure. For this purpose, let us introduce the so-called Sobol' indices [14]. The first-order Sobol' index $S_{i}, i=1, \ldots, n$ corresponds to the fraction of the variance of the output quantity (here, the instantaneous drift) that may be attributed to input variable $U_{i}$. Higher order indices $S_{i_{1}, \ldots, i_{k}}$ represent the joint effect (interactions) between variables $\left\{U_{i_{1}}, \ldots, U_{i_{k}}\right\}$. It has been shown in Sudret [15] that the Sobol' indices can be computed as a mere post-processing of the PC expansion when the latter is available.

As an example here we compute the Sobol' indices of the instantaneous drift at $t_{0}=20 \mathrm{~s}$ (Figure 5). For causality reasons this drift cannot depend on the part of the input signal that is posterior to $t_{0}$ : the corresponding indices are indeed exactly equal to zero. However, the drift does not depend either on the variables that were related to the very first time instants of the earthquake, since the corresponding indices are also equal to zero. The analysis of these indices for different time instants has shown that, whatever $t_{0}$, the instantaneous drift only depends on about 50 variables corresponding to the time instants $t_{i}$ (in the sense of Eq.(57) such that $t_{0}-5 \mathrm{~s} \leq t_{i} \leq t_{0}$. As a conclusion, each instantaneous response depends only on 50 Gaussian variables instead of the whole 500 variables (this subset of 50 variables depends of course of $t_{0}$ ). This remark helps to considerably reduce the number of basis terms considered in the LAR procedure as well as the time needed for computing the associated coefficients.

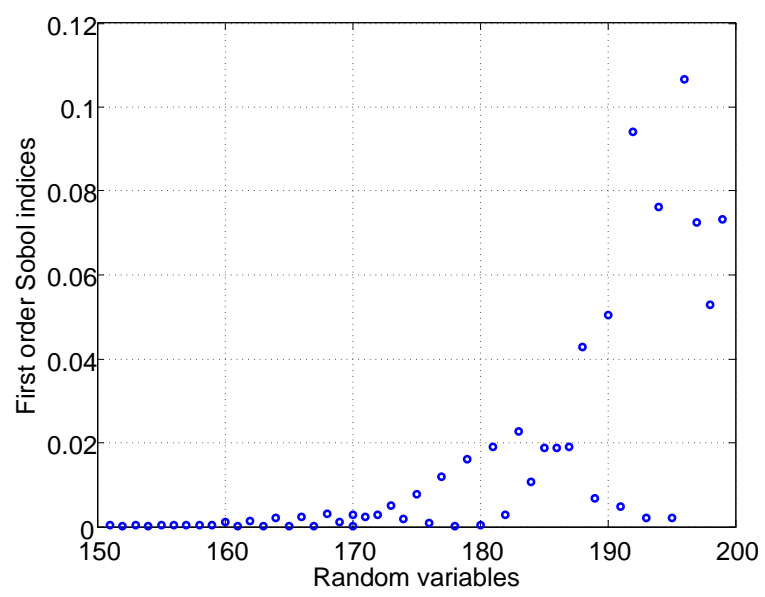

Figure 5: Sobol' indices of drift at $t_{0}=20 \mathrm{~s}$ to random variables $U_{i}, i=1, \ldots, M$

As described above (Figure 2), the range of PGA obtained through our simulations is $\left[1.3-4.3 \mathrm{~m} / \mathrm{s}^{2}\right]$, which is not large enough for establishing the fragility curves in practical applications. In order to obtain a larger range of PGA, we scaled the synthetic seismic signals by a factor $U_{s c}$ which follows a uniform distribution on the support $\mathcal{D}_{U_{s c}}=[0.1,6]$. We built a new PCE metamodel to predict the responses to scaled excitations. Due to the linear behavior of the structure, at any considered instant, the drift is expected to be the product of the scale factor $U_{s c}$ and the linear combination of Gaussian variables $U_{i}$. It means the drifts can be correctly surrogated by second order polynomial functions. 


\subsection{PCE for the drift time history}

For establishing fragility curves, we need to compute the maximal inter-storey drift $\Delta$ over time and storeys, see Eq. (9). However, this max-like quantity is not a smooth function of $\boldsymbol{U}$ and not easy to be represented by PC expansions. Indeed, the maximal interstorey drift can occur randomly at different instants during the different earthquakes. This is the reason why this quantity does not depends constantly on a given subvector of $U$ but rather on all components: no low order polynomial is capable of representing the maximal drift. As an example, using a PCE of degree 3 to predict the maximal inter-storey drift $\Delta$, we obtained the leave-one-out $Q^{2}$ estimate equal to 0.8322 , which is rather low. As an illustration, Figure 6 shows the large discrepancy between the distribution of $\Delta$ as computed from the original 1,000 samples and that obtained from the PC expansion.

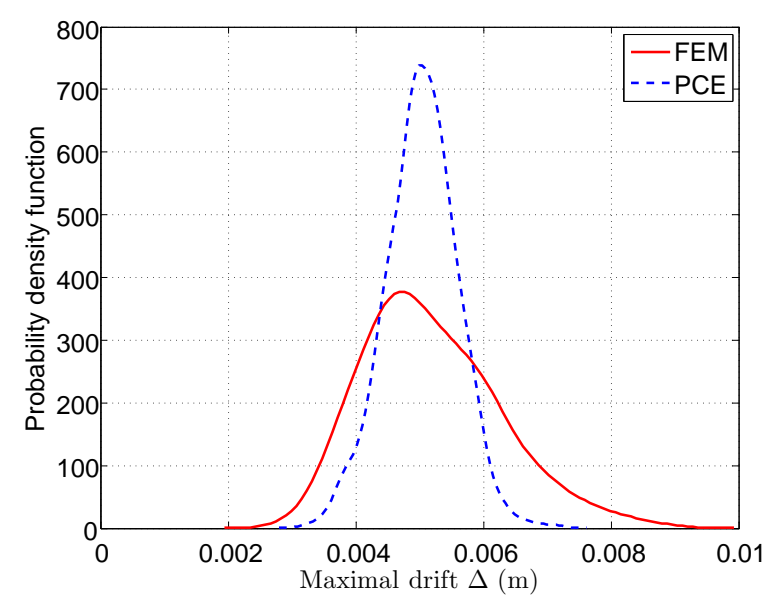

Figure 6: Maximal inter-storey drift obtained from the original Finite Element results and by a direct polynomial chaos expansion

However, PCE was shown to be efficient and accurate in predicting instantaneous inter-storey drifts. In order to obtain a metamodel of the full time-history of the structural response, we build PCE metamodels of responses at each instant during the earthquake's duration. At each instant, the PCE of the response is built from the scale factor $U_{s c}$ and the input variables $U_{i}$ within 5 seconds before it (this corresponds to 51 input variables). The accuracy of the approximation was then checked, leading to $Q^{2} \geq 0.9768$. Figure 7 compares the drifts predicted by PC expansion and the original finite element model for a particular realization of the input variables $\boldsymbol{u}_{0}$, which shows an almost perfect superposition.

To save time and memory, we considered only the strong motion phase (i.e. from $5 \mathrm{~s}$ to $30 \mathrm{~s}$ ). Unlike the intrusive approach mentioned in e.g. [9], where the PCE fails to represent the solution after a certain time because of the accumulated error in long time integration, no deterioration was detected here since PC expansions are built at each time instant separately, a feature that is only possible because of the low effective dimension of the problem (only 50 variables) and the very efficient LAR algorithm.

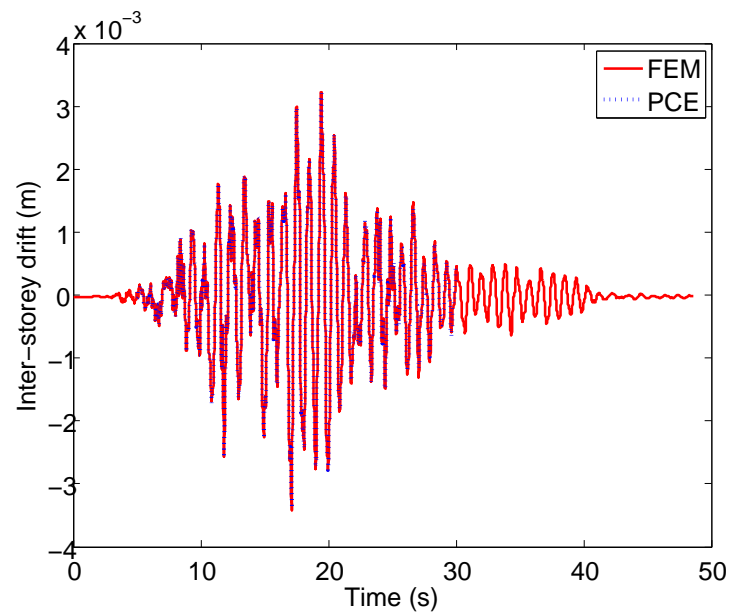

Figure 7: Time-history inter-storey drift of first floor: FE analysis vs PC expansion

\section{COMPUTATION OF FRAGILITY CURVES}

Fragility curves represent the probability of failure given the intensity of an earthquake. In this paper, it is the conditional probability that the maximal interstorey drift $\Delta$ exceeds the admissible threshold $\delta_{0}$ given PGA:

$\operatorname{Frag}(P G A)=\mathbb{P}\left[\Delta \geq \delta_{0} \mid P G A\right]$

\subsection{Classical approach}

The classical approach to establish fragility curves consists in assuming a lognormal shape for the curves. More specifically, the maximal inter-storey drift $\Delta$ is modeled by the lognormal distribution in which the $\log$-mean value $\lambda$ is a linear function of PGA:

$$
\ln \Delta \sim \mathcal{N}(\lambda, \zeta) \quad \lambda=A \ln (P G A)+B
$$

Parameters $A$ and $B$ are determined by means of linear regression in a log-log plot, as shown in Figure 8 . The same approach is widely applied in the literature, see e.g. Choi et al. [5], Padgett and DesRoches [12] among others. In this study, the coefficient of determination $R^{2}$ of linear regression is 0.95 . The standard deviation $\zeta$ is evaluated in accordance with its definition [10]. Let us denote by $e_{i}$ the residual between the actual value of the dependent variable and the value predicted by the linear model: $e_{i}=\ln \Delta_{i}-A \ln \left(P G A_{i}\right)-B$. Then we obtained $\zeta^{2}=\sum_{i=1}^{m} e_{i}^{2} /(m-2)$ with $m=1,000$ observations. In the present case, we obtained the following values for the parameters: $A=0.9784, B=-7.1585$ and $\zeta=0.1994$. by:

The probability of failure in Eq. (9) is then given

$$
\begin{aligned}
\operatorname{Frag}(P G A) & =\mathbb{P}\left[\ln \Delta \geq \ln \delta_{0}\right]=1-\mathbb{P}\left[\ln \Delta \leq \ln \delta_{0}\right] \\
& =1-\Phi\left(\frac{\ln \delta_{0}-[A \ln (P G A)+B]}{\zeta}\right)
\end{aligned}
$$




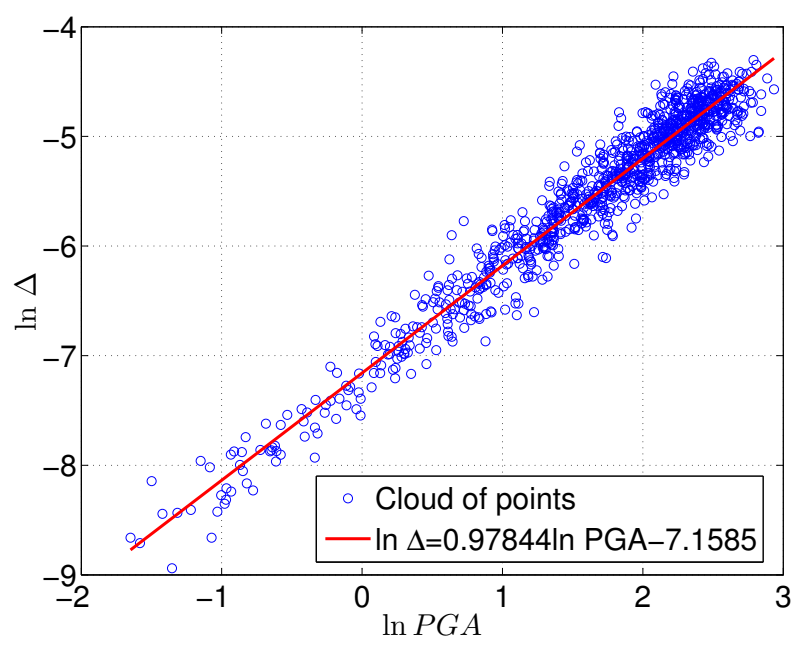

Figure 8: Cloud of 1,000 data pairs $(\log P G A, \log \Delta)$

where $\Phi(t)=\int_{-\infty}^{t} e^{-u^{2} / 2} / \sqrt{2 \pi} \mathrm{d} u$ is the Gaussian cumulative distribution function. Figure 9 depicts the fragility curves associated to the different values of $\delta_{0}$.

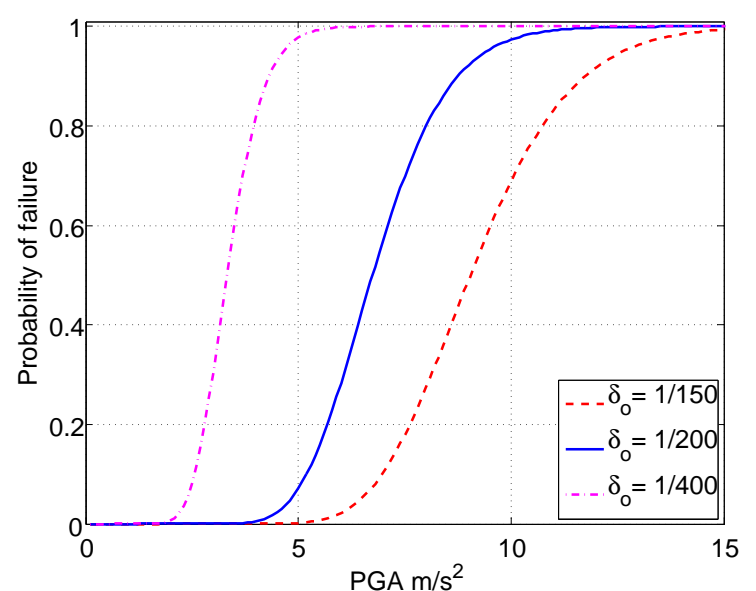

Figure 9: Fragility curves obtained by the lognormal approach

\subsection{Empirical approach}

Having at hand a large sample set of pairs $\left\{\left(P G A_{j}, \Delta_{j}\right), j=1, \ldots, N\right\}$, it is possible to use a non parametric, so-called empirical approach to compute the fragility curve. Let us consider a given abscissa $P G A_{o}$. Within a small bin surrounding $P G A_{o}$, say $\left[P G A_{o}-h, P G A_{o}+h\right]$ one assumes that the maximal drift $\Delta$ is linearly related to the PGA. (Note that this assumption is exact in the case of linear structures but would be only an approximation in the non linear case). Therefore, the maximal drift $\Delta_{j} \in\left[P G A_{o}-h, P G A_{o}+h\right]$ related to $P G A_{j}$ is converted to the drift $\Delta_{o}$ which would be related to a similar input signal having a peak ground acceleration of $P G A_{o}$ as follows:

$\Delta\left(P G A_{o}\right)=\Delta_{j} \frac{P G A_{o}}{P G A_{j}}$
The value of the fragility curve at $P G A_{o}$ is obtained by a crude Monte Carlo estimator:

$\widehat{\operatorname{Frag}}\left(P G A_{o}\right)=\frac{N_{f}\left(P G A_{o}\right)}{N_{s}\left(P G A_{o}\right)}$

where $N_{f}\left(P G A_{o}\right)$ is the number of observations in the vicinity of $P G A_{o}$ such that $\Delta\left(P G A_{o}\right) \geqslant \delta_{o}$ and $N_{s}\left(P G A_{o}\right)$ is the total number of observations that fall into the bin $\left[P G A_{o}-h, P G A_{o}+h\right]$.

The vicinity is defined by the bin width $2 h$, which is selected according to the sample set of observations. In this study, $h=0.1 \mathrm{~m} / \mathrm{s}^{2}$ is chosen. This empirical approach is close to the incremental dynamic analysis (IDA) in Vamvatsikos and Cornell [16], except for this: while using IDA, the failure probability at a given $P G A_{o}$ is obtained from analyses of excitations with the exact $P G A_{o}$, due to the rescaling of the signals. The data post-processing on the $10^{5}$ observations leads to the fragility curves in Figure 10 . We also established the curves using only 20,000 samples. Figure 10 shows that resulting curves are consistent with the curves from 100,000 samples even for the low probability of failure.

As a summary, although they are prone to some statistical noise, the curves established by this empirical approach are stable provided that a sufficient number of observations in each interval is available. This is only possible when using a large sample set (e.g. $N=10^{5}$ ) which can only be carried out when a surrogate model is used.

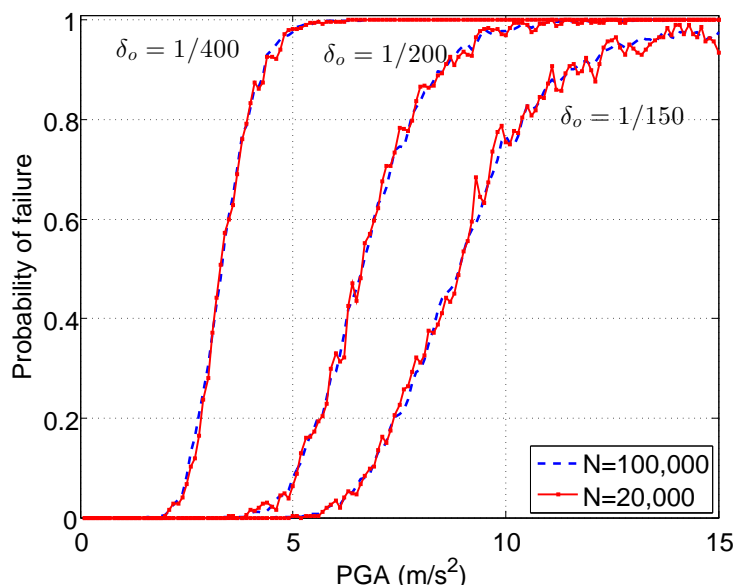

Figure 10: Fragility curves by empirical approach using $\mathrm{N}=100,000$ and $\mathrm{N}=20,000$ samples

The curves obtained by the classical and the PC expansion-based approaches are compared in Figure 11. Due to the accuracy of the PC expansion, the fragility curves based on a large sample $(100,000)$ evaluated by PCE can be considered a reliable benchmark. The "classical" fragility curve obtained under the lognormal assumption is then validated in the case of the elastic structure using the polynomial chaos surrogate and a non parametric representation of the results. One should remark that the lognormal curve becomes slightly less accurate for the more severe level of damage. 


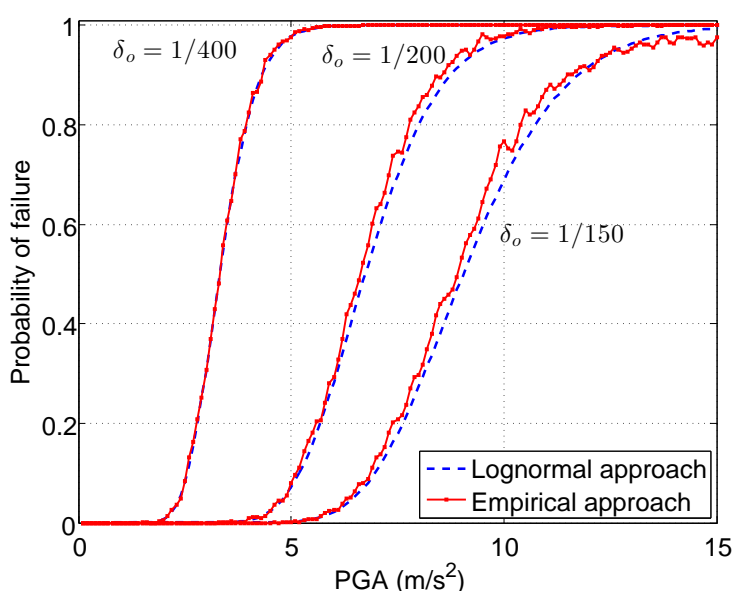

Figure 11: Comparison of fragility curves from empirical and lognormal approach

\subsection{Modified lognormal approach}

Due to the linearity of the system, we can use the following strategy to compute the conditional probability in Eq. (9). The seismic signals are scaled to get a PGA that is equal to a reference value, e.g. $P G A_{\text {ref }}=1 \mathrm{~m} / \mathrm{s}^{2}$ ( the choice of $P G A_{\text {ref }}$ does not affect the final curves). The corresponding maximal drifts are also consistently scaled:

$\Delta(P G A)=\Delta_{r e f} \frac{P G A}{P G A_{r e f}}$

Eq. (9) becomes:

$\widehat{\operatorname{Frag}}(P G A)=\mathbb{P}\left[\frac{P G A \Delta_{\text {ref }}}{P G A_{\text {ref }}} \geq \delta_{0}\right]$
$=\mathbb{P}\left[\Delta_{r e f} \geq \frac{P G A_{r e f}}{P G A} \delta_{0}\right]=1-F_{\Delta_{r e f}}\left(\frac{P G A_{r e f}}{P G A} \delta_{0}\right)$

If we assume that the maximal inter-story drift $\Delta_{r e f}$ at $P G A_{\text {ref }}$ has a lognormal distribution as in Eq. (10), then Eq. (15) becomes:

$$
\widehat{\operatorname{Frag}}(P G A)=1-\Phi\left(\frac{\ln \left(\frac{P G A_{r e f}}{P G A} \delta_{0}\right)-\mu_{\ln \Delta_{r e f}}}{\sigma_{\ln \Delta_{r e f}}}\right)
$$$$
=1-\Phi\left(\frac{\ln \delta_{0}-\left(\ln P G A+\mu_{\ln \Delta_{r e f}}-\ln P G A_{r e f}\right)}{\sigma_{\ln \Delta_{r e f}}}\right)
$$

where $\mu_{\ln \Delta_{r e f}}$ and $\sigma_{\ln \Delta_{r e f}}$ are respectively the mean value and standard deviation of the random variable $\ln \Delta_{r e f}$. Comparing Eq. (11) and (16), one can observe that they are fully consistent provided $A=1$, $B=\mu_{\ln \Delta_{r e f}}-\ln P G A_{r e f}=-7.1946, \zeta=\sigma_{\ln \Delta_{r e f}}=$ 0.2005 .
We can then derive the fragility curves using the lognormal approach with parameters evaluated by Eq. (16) and a limited number of observations $(1,000$ in this case). The resulting curves are more consistent with the empirical ones than the original lognormal curves obtained by regression.

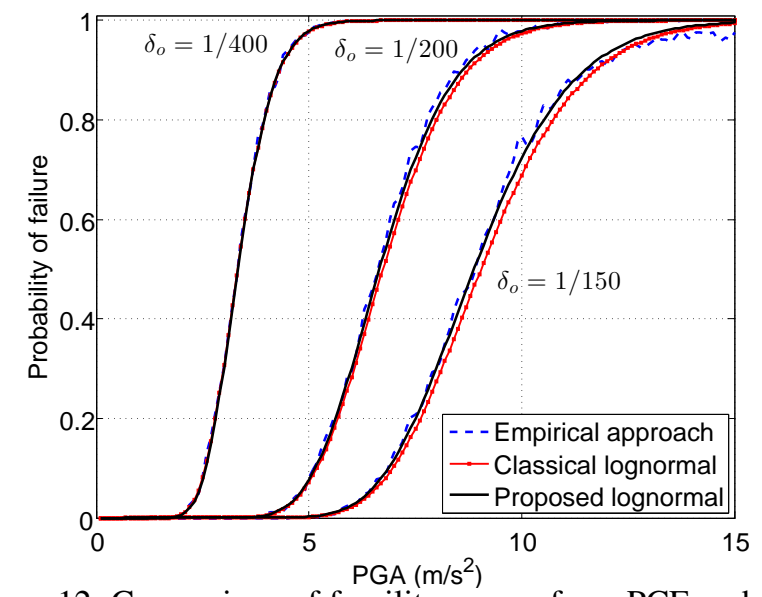

Figure 12: Comparison of fragility curves from PCE and modified lognormal approach

The proposed approach takes advantage of the linear behavior in the model, which imposes the unit value $A=1$. Note that Cornell et al. [6] also empircally propose to take this parameter equal to 1 .

\section{CONCLUSION \& PERSPECTIVE}

In this paper, polynomial chaos expansions are introduced in the framework of seismic probabilistic risk assessment to compute the fragility curves of a structure under earthquake excitation.

Due to the high computational cost, the current practice relies on a limited number of transient finite elements analyses as well as a strong assumption on the shape of fragility curves, e.g. a lognormal cumulative distribution function. Polynomial chaos expansions surrogates the responses of interest, then allow the computation of responses to numerous excitations without running new finite elements analyses.

We proved the accuracy of the PCE approach in predicting the time-history of the inter-story drift of a linear frame structure subject to seismic excitations with similar spectral and temporal contents. The comparison between PCE-based and "classical" fragility curves showed that the assumption on lognormal shapes is correct for this type of structure. Moreover, the linear regression technique applied to determine the parameters of lognormal curves leads to accurate fragility curves in particular for slight damage. We also proposed a new manner for estimating parameters of the lognormal curves which makes the resulting curves more consistent with the MCS-based ones.

However, due to the fact that the PCE of every instantaneous response needs to be computed, PCE approach remains costly. Principal Component Analysis [4] may provide a suitable solution to reduce 
the computational costs involved. Further improvements of the method are currently investigated. First the spectrum of synthetic signals shall be enlarged for real application without using a scaling factor. This can be carried out by randomizing the 6 parameters $\left\{\alpha_{1}, \alpha_{2}, \alpha_{3}, \omega_{m i d}, \omega^{\prime}, \zeta_{f}\right\}$. This work is currently in progress.

\section{REFERENCES}

[1] Berveiller, M., B. Sudret, and M. Lemaire (2004). Presentation of two methods for computing the response coefficients in stochastic finite element analysis. In Proc. 9th ASCE Specialty Conference on Probabilistic Mechanics and Structural Reliability, Albuquerque, USA.

[2] Blatman, G. and B. Sudret (2010). An adaptive algorithm to build up sparse polynomial chaos expansions for stochastic finite element analysis. Prob. Eng. Mech. 25(2), 183-197.

[3] Blatman, G. and B. Sudret (2011a). Adaptive sparse polynomial chaos expansion based on Least Angle Regression. J. Comput. Phys 230, 23452367.

[4] Blatman, G. and B. Sudret (2011b). Principal component analysis and Least Angle Regression in spectral stochastic finite element analysis. In M. Faber (Ed.), Proc. 11th Int. Conf. on Applications of Stat. and Prob. in Civil Engineering (ICASP11), Zurich, Switzerland.

[5] Choi, E., R. DesRoches, and B. Nielson (2004). Seismic fragility of typical bridges in moderate seismic zones. Engineering Structures 26(2), 187 -199 .

[6] Cornell, C.-A., F. Jalayer, R. Hamburger, and D. Foutch (2002). Probabilistic basis for 2000 SAC federal emergency management agency steel moment frame guidelines. J. Struct. Eng. (ASCE) 128(4), 526-533.

[7] Cornell, C.-A. and P. Krawinkler (2000). Progress and challenges in seismic performance assessment. Technical report, PEER Center, USA.

[8] Efron, B., T. Hastie, I. Johnstone, and R. Tibshirani (2004). Least angle regression. Annals of Statistics 32, 407-499.

[9] Le Maitre, O., L. Mathelin, O. Knio, and M. Hussaini (2009). A synchronous time integration for polynomial chaos expansion of uncertain periodic dynamics. Preprint.

[10] Mai, V. (2012). Establish fragility curves of buildings subject to seismic excitation. Master's thesis, Ecole des Ponts ParisTech.
[11] Pacific Earthquake Engineering and Research Center (2004). OpenSees: The Open System for Earthquake Engineering Simulation.

[12] Padgett, J.-E. and R. DesRoches (2008). Methodology for the development of analytical fragility curves for retrofitted bridges. Earthquake Eng. Struct. Dyn. 37(8), 1157-1174.

[13] Rezaeian, S. and A. Der Kiureghian (2010). Simulation of synthetic ground motions for specified earthquake and site characteristics. Earthquake Eng. Struct. Dyn. 39(10), 1155-1180.

[14] Sobol', I. (1993). Sensitivity estimates for nonlinear mathematical models. Math. Modeling \& Comp. Exp. 1, 407-414.

[15] Sudret, B. (2008). Global sensitivity analysis using polynomial chaos expansions. Reliab. Eng. Sys. Safety 93, 964-979.

[16] Vamvatsikos, D. and C.-A. Cornell (2002). Incremental dynamic analysis. Earthquake Eng. \& Struct. Dyn. 31(3), 491-514.

[17] Zareian, F. and H. Krawinkler (2007). Assessment of probability of collapse and design for collapse safety. Earthquake Eng. Struct. Dyn. 36(13), 1901-1914.

[18] Zentner, I. (2010). Numerical computation of fragility curves for NPP equipment. Nuc. Eng. Des 240, 1614-1621. 